\title{
Transcranial magnetic stimulation in a male brain and his genitals for the influence of his genital system with problems related to his spermatozoa mobility and density
}

\begin{abstract}
Studies on a case of a woman that experienced many miscarriages without having any serious gynecological problems led us to search as an alternative to examine in a microbiological diagnostic laboratory her husband's genital system. This test detected problems in man's genital system. To overcome these problems, we thought of applying weak magnetic fields of the order of pT-TMS (pico-Tesla Transcranial Magnetic Stimulation) on man's brain and genital system in order to influence his genital system for its proper function with respect to the movement, the density and the better penetration of the spermatozoa after ejaculation in his wife ovule.
\end{abstract}

Keywords: MEG, pT-TMS, spermatozoa, man genitals
Volume 12 Issue 2 - 2021

\author{
Photios Anninos, ' Nikolia Anninou, ${ }^{2}$ Adam \\ Adamopoulos,' 'Nikolaos Tsagas ${ }^{3}$ \\ 'Laboratory of Medical Physics, Department of Medicine, School \\ of Health Sciences, Democritus University of Thrace, University \\ Campus, Alexandroupoli, Greece \\ 2Private Sector Veterinarian, Alexandroupolis, Greece \\ ${ }^{3}$ Department of Electrical Engineering, Engineering Faculty, \\ Democritus University of Thrace, Xanthi, Greece
}

Correspondence: Photios Anninos, Emeritus Professor,

Laboratory of Medical Physics, Department of Medicine, School of Health Sciences, Democritus University of Thrace, University Campus, Alexandroupoli, Greece,

Email pans.photios.anninos@gmail.com

Received: April 16, 2021 | Published: April 26, 202

\section{Introduction}

In general, the man spermatozoa consist from the head, the main body and the tail. The head has diameter $3.4-5 \times 10^{-6} \mathrm{~m}$ in length and transversal diameter $1.5-2.8 \times 10^{-6} \mathrm{~m}$. The volume of the head calculated electronically is $15------29 X\left(10^{-6} \mathrm{~m}\right)^{3}$ and the main body of the spermatozoa consists from the neck and the medium section. The neck has length about $1 \times 10^{-6} \mathrm{~m}$ and the medium section $5--7 \times 10^{-6} \mathrm{~m}$ and diameter $1 \times 10^{-6} \mathrm{~m}$.

The tail of the spermatozoa consists from the main part with length about $45 \times 10^{-6} \mathrm{~m}$ and the final section of the tail has length $5-7 \times 10^{-6} \mathrm{~m}$.

The head of the spermatozoa is covered from the acrosoma where is riched with phospholipidia, glicoproteins and different enzymes and is covered with internal and external cloak. The best enzyme of this space is the acrosini which is important for the penetration of the spermatozoa in the woman ovule. The head of the spermatozoa has special figure to be able to go through the liquids. The movement of the spermatozoa is based on the mechanisms of the micropipes. In the region of the middle section of the spermatozoa is covered with mitochondria which give energy for the movement of the spermatozoa.

The Sperma is characterized from the liquid mass which comes out from the man urethra during the ejaculation. The Sperma consists from the spermatozoa and the sperma plasma. These two are not homogeneous immediately after ejaculation. The more part of the cells of normal sperma is the spermatozoa which are motion cells with head, with cell body and tail. On the head of the spermatozoa are sit upon in the form mantile the acrosoma which consists from different enzymes as is the acrosini. In the acrosoma there are different enzymes as is the glycosidase and others. The acrosoma plays important role for the penetration of the sperma in the woman ovule. In order the spermatozoa to penetrate the woman ovule it is necessary the chemical change in the cell membrane and after the changes in the actosoma. In the physiological state of the spermatozoa $90-95 \%$ has acrosoma after the ejaculation. In case that the spermatozoa are out from the somatic plasma then $15 \%$ of the spermatozoa loss the acrosoma and the power to penetrate the woman ovule. The middle part of the spermatozoa consists from the axonima which has around it the mitochondria. The mitochondria create a lot of energy which is needed for the movement of spermatozoa. The tail and the inside of it axonima and the two central and nine external micronimatia produce the great movement of the spermatozoa which is the proper power for the fertilization. The best movement of the spermatozoa from the biochemical point of view depends from the neucleotide (ATP).The density in ATP goes with the movement magnitude of the spermatozoa and the duration of the movement.

In addition to the energy from mitochondria there are others substances as electrolytes, potassium, spermatozoa. If the speed of the spermatozoa is $30-100 \mu \mathrm{m} / \mathrm{sec}$ is possible to approach the woman ovule, which is $15 \mathrm{~cm}$ away, in one hour after the ejaculation. The physiological number of spermatozoa during the ejaculation is $10-120$ milions $/ \mathrm{ml}$ for fertilization. From this only $50 \%$ are active whereas the $20 \%$ developed problems of their membranes and only $40 \%$ of the spermatozoa don't have physiological forms. In the spermatozoa there are also spermatides which don't have acrosoma.

There is other kind of cells of spermatozoa the epithelial cells which comes from the different parts of the discharged way of spermatozoa. Other cells in the sperma in case of inflammation are the kokkiocells and lymphocells. There is also amino acid in the plasma of the spermatozoa. In the amino acid Karnitine which is in $2 \mathrm{SO} \mu \mathrm{g} / \mathrm{ml}$ in normal spermatozoa if then after cutting the spermatic passage then the karnitine is lowered very much the spermatic plasma. There are also concentrations of proteins in the spermatozoa plasma in $58 \mathrm{~g} / \mathrm{I}$. 
Most of the proteins are enzymes and after the ejaculation was seen coagulation of the spermatozoa and after $20-30 \mathrm{~min}$ is liquefied the spermatozoa with one enzyme the seminin. The perforation of the cell membrane of the woman ovule comes from the acrosini of the spermatozoa. In the plasma of the spermatozoa there are lipidia as the sperminiand prostaglandins. In the plasma of the spermatozoa there are also testosterone and cortizoli and FSH and prolactine which are important for the movement of the spermatozoa. In the spermatozoa and other substances of the plasma can create antibodies which are very important for immunological reactions.

\section{Methods}

Studies with the use of weak magnetic fields ${ }^{2-8}$ in patients with different brain disorders and their effect we have thought to influence the man genitals system for the proper function with respect to the movement, the density and the better penetration of the spermatozoa after ejaculation in the woman ovule.

In order to improve the proper function of the spermatozoa which we have mentioned above ${ }^{1}$ so that properly penetrate the woman's ovule after ejaculation we have thought to do first magnetoencephalographic (MEG) measurements using the biomagnetometer SQUID in our laboratory for the man with genital problems. After getting the MEG

Table I Microbiological diagnostic findings before and after treatment from aimoanalysi Ebrou

\begin{tabular}{|c|c|}
\hline Microbiological diagnostic findings before & Microbiological diagnostic findings after \\
\hline Treatment from aimoanalysi Ebrou & Treatment from aimoanalysi Ebrou \\
\hline June 23,2020 & November 4,2020 \\
\hline Volume sperma before & Volume sperma after \\
\hline $2.0 \mathrm{ml} \mathrm{NV}>2 \mathrm{ml}$ & $1.5 \mathrm{ml} \mathrm{NV}>2 \mathrm{ml}$ \\
\hline Reaction(PH)7.5 NV>2ml & Reaction(PH)7.2 \\
\hline Head with head litle & Head with head litle \\
\hline Tail with head rare & Tail with head rare \\
\hline Tail with tail rare & Tail with tail rare \\
\hline Mixtures more & Mixtures litle \\
\hline Different shapes $7 \% \mathrm{NV}>14$ & Different shapes I5\% NV>14 \\
\hline Abnormals head $42 \%$ & Abnormals head $36 \% \mathrm{NV}>14$ \\
\hline Abnormal neck $30 \%$ & Abnormal neck $31 \% \mathrm{NV}>14$ \\
\hline Abnormal tails $10 \%$ & Abnormal tails $10 \% \mathrm{NV}>14$ \\
\hline Immature II\% & Immature $8 \%$ \\
\hline Fast Motion in front $\mathrm{Il} \%$ in $\mathrm{Ih}$ & Fast Motion in front $25 \%$ in $\mathrm{Ih}$ \\
\hline Fast Motion in front in $6 \%$ in $3 \mathrm{~h}$ & Fast Motion in front $15 \%$ in $3 \mathrm{~h}$ \\
\hline Sluggish in front $15 \%$ in $\mathrm{Ih}$ & Sluggish in front $20 \%$ in Ih \\
\hline Sluggish in front $5 \%$ in $3 \mathrm{~h}$ & Sluggish in front I $5 \%$ in $3 \mathrm{~h}$ \\
\hline Local motion $25 \%$ in $\mathrm{Ih}$ & Local motion $30 \%$ in $\mathrm{Ih}$ \\
\hline Local motion $10 \%$ in $3 \mathrm{~h}$ & Local motion $25 \%$ in $3 \mathrm{~h}$ \\
\hline No motion $49 \%$ in Ih & No motion $25 \%$ in Ih \\
\hline No motion $79 \%$ in $3 \mathrm{~h}$ & No motion $45 \%$ in $3 \mathrm{~h}$ \\
\hline
\end{tabular}

Citation: Anninos P,Anninou N,Adamopoulos A, et al.Transcranial magnetic stimulation in a male brain and his genitals for the influence of his genital system with problems related to his spermatozoa mobility and density. Obstet Gynecol Int J. 202 I;12(2):I03-105. DOI: I0.15406/ogij.202I.I2.00560

recordings of the patient we have to do Fast Fourier Transform (FFT) statistical analysis in order to determine his alpha frequency (813HZ). In addition, we have to examine after the ejaculation of the patient the movements and the density of spermatozoa in diagnostic microbiological laboratory.

Then, after having determined the patient alpha frequency we can calibrate the electronic device ${ }^{9}$ in that frequency and advise the patient to use the device every night at the $23.30 \mathrm{pm}$ hour for one minute in frontal and occipital lobes of his head, and another minute for the right and left temporal regions of the patient head. After we have to advise the patient to turn off all the lights and go to bed to sleep. In addition, the patient has to use the electronic device ${ }^{9}$ for the treatment some time at noon, for his genitals organs for one minute every day. Furthermore, the patient has to do the above mention treatments for about a month and go for a diagnostic microbiological laboratory to analyze his spermatozoa after ejaculation. These spermatozoa conditions should to compare with previous results before and after treatment (Table 1) (Table 2). The same procedure the patient repeats for another month and if he has proper results from the diagnostic microbiological laboratory he should visit his gynecology medical doctor for his/her advise for his contacts with his wife in order to see if she can get pregnant. After his wife with the above treatment become pregnant we can see the progress of the fetus every week. 
Table 2 Microbiological diagnostic findings after treatment July 29, 2020

\begin{tabular}{l}
\hline Protypo diagnostic laboratory Demokritos \\
\hline Volume sperma \\
Volume $2.0 \mathrm{ml} \mathrm{NV} 1.5-5.0$ \\
Reaction(PH) $7.7 \mathrm{NV}>7.2 \mathrm{ml}$ \\
Liquefied $60 \mathrm{NVI} 5-45$ \\
Number of sperma/mI 23 \\
NV>I5XI06 \\
Number of sperma total 46 \\
NV >39XI06 \\
Nomal forms $4 \% \mathrm{NV}>=4 \%$ \\
Abnormal heads $65 \%$ \\
Abnormak tails II $\%$ \\
Fast Motion in front $10 \%$ in Ih \\
Fast Motion in front $8 \%$ in $3 \mathrm{~h}$ \\
Slow Motion I $2 \%$ in Ih \\
Slow motion $10 \%$ in $3 \mathrm{~h}$ \\
Sluggish motion $10 \%$ in Ih \\
Sluggish motion $9 \%$ in $3 \mathrm{~h}$ \\
No motion $68 \%$ in Ih \\
No motion $73 \%$ in $3 \mathrm{~h}$ \\
\hline
\end{tabular}

\section{Discussion}

In our studies with continuum many miscarriages of the woman without having any serious gynecological problems we thought to examined in a microbiological diagnostic laboratory her husband's genital system. With that examination we were able to find out problems in his genital system. In order to overcome these problems we thought of using weak magnetic fields of the order of pT-TMS (pico-Tesla Transcranian Magnetic Stimulation) ${ }^{2-8,9}$ in her husband's brain and genital system so that to be able to influence his genital system for its proper function with respect to the movement, the density and the better penetration of the spermatozoa after ejaculation in his wife ovule.

Of course further research is needed to be done in order to clarify our research results.

\section{Results}

After the application with pico-TMS 9 in the different parts of her husband brain and genital system with the proper alpha frequency, obtained using FFT in the MEG recordings, we have to examine, after his ejaculation, the movements and the density of spermatozoa in a diagnostic microbiological laboratory as we have mentioned in our Methods.

By comparing these spermatozoa conditions before and after the external magnetic stimulation we have the results which are shown in Table 1 for the (for June 23, 2020 and, November 4, 2020) and from another microbiological laboratory for July 29, 2020 in Table 2. The same procedure we have advised her husband to repeat for another month and after has proper results from the same microbiological laboratory he should visit his gynecology medical doctor for his/her advise for his contacts with his wife if she could get pregnant with these spermatozoa conditions.

Thus, if his wife with the above treatment becomes pregnant after so many miscarriages, we can see the normal progress of the fetus every week from microbiological diagnostic laboratory.

\section{Conclusion}

Using continuously the application of external pico-Tesla Transcranial Magnetic Stimulation on the different parts of the brain and genitals for the man who his wife was suffering with many miscarregies, she finaly became pregnant.

\section{Acknowledgments}

None.

\section{Author's contribution}

All the authors have accepted responsibility by the entire content of this manuscript and approved submission.

\section{Funding}

This research was funded by the partnership between GGET (General Secretariat of Research and Technology, GR) and ERGO AEBE, INC, GR (Grant Number: 80623).

\section{Conflicts of interest}

The authors declare no conflict of interest.

\section{References}

1. Papadopoulos H. Pathology of man genital system. In Greek Medical publishing company, ZHTA; 1994.

2. Photios A, Nicolia A, Adam A, et al. We Used a Double Blind Experiment to Investigate Weak pT-TMS in Epilepsy Patients. Maedica (Bucur). 2020;15(1):92-95.

3. Anninos P, Kotini A, Adamopoulos A, et al. MEG recordings of patients with cerebral palsy before and after the application of pico-Tesla weak magnetic fields. J Integr Neurosci. 2019;18.1:17-21.

4. Anninos P, Adamopoulos A, Kotini A, et al. MEG study of picotesla trancranial magnetic simulation on patients with depression. EC Neurology. 2017;5.3:115-122.

5. Anninos P, Adamopoulos A, Kotini A, et al. MEG evaluation of pico-Tesla external TMS on multiple sclerosis patients. Mult Scler Relat Disord. 2016;8:45-53.

6. Anninos P, Adamopoulos A, Kotini A, et al. Combined MEG and pT-TMS study in Parkinson's disease. J Integr Neurosci. 2016;15(2):145-162.

7. Anninos P, Adamopoulos A, Anninou N, et al. The proper function of pineal and thymus glands to control foreign organisms actign on human brain subjects. EC Neurology. 2020;12(6):59-63.

8. Photios A, Nicolia A, Adam A, et al. Oral intake of melatonin hormone could influence the thymus gland for producing the proper antibodies. EC Neurology. 2020;12(8):175-176.

9. Anninos P, Tsagas N. Electronic apparatus for treating epileptic individuals. USA patent; 1995:5453072. 